\title{
DIGITAL DETAILING ON THE EDGE: JOINING OF COMPLEX CURVED COMPOSITE PANELS
}

\section{DETALHAMENTO DIGITAL NO LIMITE: JUNTAS DE PAINÉIS COMPOSTOS COM CURVATURAS COMPLEXAS}

\section{Andre Chaszar}

Principal, O-Design Research \& Consulting, ac@0-design.com

\begin{abstract}
This paper concerns the detailing of panel edge joints within the context of an adaptive subdivision system for composite panelling of complex curved surfaces. A general overview of this context is provided, followed by more in-depth description of the challenges of joining the resulting panels as well as various alternatives for doing so. The alternatives are evaluated with respect to multi-criterion considerations including panel materials, machining capabilities, joint forces, joint orientation, appearance and weather-resistance among others, which are expected to vary from project to project - as well as possibly among locations within a project - and therefore affect the choice(s) of detailing.
\end{abstract}

Keywords: architectural geometry, CNC fabrication, multi-criterion optimisation, skin-and-core ('sandwich') composites.

\section{Introduction - digital technology, complex geometry, performance, making choices}

Digital technologies evolving over the past few decades have been offering new opportunities for the design, analysis and fabrication of architectural artifacts in ways which can both resemble (even imitate) but also differ from formerly developed and implemented ways of doing so. The following gives an overview of various joint detailing approaches and options arising from the need to connect adjacent panels forming a complex curved surface, and it describes and analyses the role(s) that digital technologies play in various facets of the designanalysis-fabrication process. Most importantly, perhaps, it highlights the potential for (re-)integration of these subprocesses (CHASZAR, 2006), which during much of the 20th century and even earlier stages of the Industrial Revolution tended to become separated from each other in consequence of the specialisation trend and other socio-economic forces. This reintegrative potential offers not only increased opportunity for proposal and testing of innovative designs and techniques, but also for rediscovery and adoption or adaptation of earlierdeveloped (physical, representational and behavioral) patterns and methods.

The topic of detailing as influenced by computation is also interesting for other reasons including the tensions between speed, precision, reliability and care which arise as automated processes of decision and production confront human needs for involvement and attachment
(SENNETT, 2008). While computation-based techniques enable dealing with ever larger amounts of information at ever greater speeds, the outcome of the race between these capabilities and the desire for greater variation and complexity is as yet undecided, even setting aside the distancing effects inherent in working with abstractions.

Turning now to the more specific case before us: joint detailing choices are typically made after establishment of joints' quantity, locations and orientations, although some revision to these more 'global' choices may be undertaken consequently to ('local') detailing choices (CHASZAR, 2004) resulting in a possibly recursive process which may or may not converge ${ }^{1}$. (See Figure 1.) The different joint detailing choices already available and new options perhaps developable typically need to be evaluated and compared with each other on the basis of various criteria pertaining to qualitative and quantitative aspects, often referred to as 'performance' ${ }^{2}$. These aspects include ideal/abstract and practical/concrete factors, short- and long-term ones, private and public, etc., covering a great (potentially limitless) range and depth of requirements. Normally limitations of time and budget as well as knowledge and other biases (implicit) or preferences (explicit) of the project team - including designers of various disciplines, builders, facility owners and sometimes even occupants/users - result in substantially fewer than the full possible complement of relevant factors being taken into account. This leaves ample room for flexibility in decision-making, although at the expense of strict rationality. In any case, techniques 

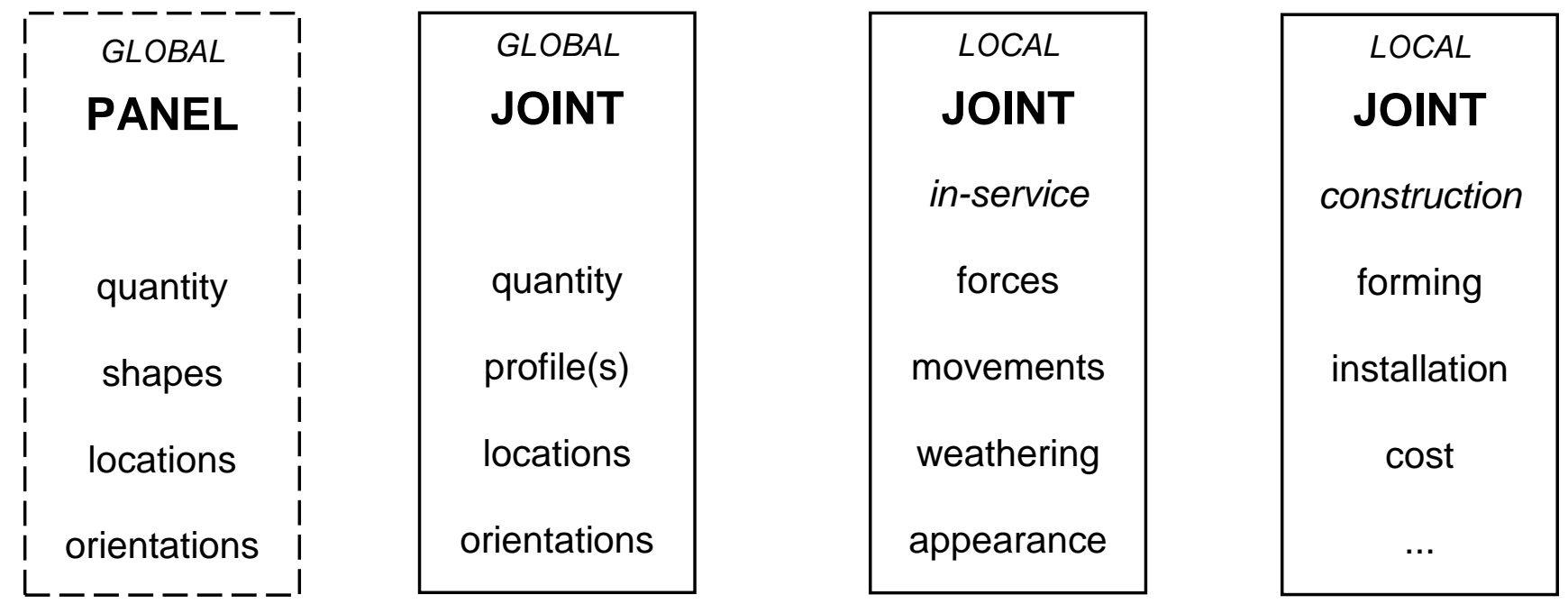

Figure 1. Classification of some important joint detailing parameters and criteria - global vs. local, in-service vs. construction, etc. Note that there is no definite hierarchy among these, but instead recursion and bi-directionality are common. Nor can any list be exhaustive, all factors contributing to the 'ill-defined' or even 'wicked' nature of the design task. Source: author, 2013.

of 'multi-criterion optimisation' (MCO) and/or 'multicriterion decision analysis' (MCDA) are applicable in generating, evaluating, comparing and selecting options.

It should be borne in mind, though, that the multiplicity of potentially conflicting (if not outright contradictory) aspects and therefore goals (or 'sub-goals') means that a clear optimum is unlikely to be found, not only because the 'search space' is too large, but also because this clear optimum is unlikely to exist. Therefore, various 'suboptimal' choices may be found to be roughly equally good overall - considering their various pros and cons so that some other 'external' criterion or basis for choice must be introduced. These observations apply to almost any object of design, of course, but are pointed out here as a reminder and to highlight the possibly arbitraryseeming nature of the outcomes of complex jointdetailing situations, which remain to some extent therefore always open to question and argument. This stands in contrast with simpler design tasks - sometimes referred to as 'tame' or 'well-defined' problems, as opposed to 'wicked' or 'ill-defined' ones (RITTEL \& WEBBER, 1973) - where there is general, if not complete, agreement regarding the characteristics of a correct outcome and the procedures for reaching one. Thus, the many trade-offs inherent in designing and choosing joint details mean it is not possible to validly address the situation with linear procedures and unchanging goals.

A question - perhaps more academic than practical may arise regarding just how much detailing (of panel joints or more generally) differs with the introduction of digital techniques and digital practice. Is the impact revolutionary - a step-change in the means and ends of detailing - or rather incremental? The answer is: 'quite a lot'/revolutionary or 'hardly at all'/incremental, depending on where we focus our attention. On the 'quite a lot' side fall indirect factors such as 1) the potential exploration of a much larger number and variety (thus 'space') of detailing 'solutions' enabled by digital-computational techniques including performance simulations and MCO/MCDA, and 2) the delegation of much repetitive design work (e.g. surface subdivision) to algorithms, as well as direct factors like 3 ) the potentially greater quantity and/or complexity of forming operations achievable with CNC techniques. However, 'hardly at all' encompasses any number of constraints both physical and social, including for example the continued invariance of the many relevant physical and chemical processes (e.g. deformation, capillary action, corrosion, etc.) and the human processes of motivation and negotiation which condition what is actually achievable in a given time and place (since full automation of design, review/permitting, fabrication, and assembly is neither desirable nor feasible within the foreseeable future). Thus, while complex geometries are in some respects more tractable with computational techniques, digital (virtual) detailing is still heavily beholden to analog (real) constraints.

Given the foregoing observations on some of the main themes at play, we can now turn to more specifics of the situation at hand: the context of panelising complex curved surfaces and the particular details of joining panels.

\section{Context - subdivided complex curved surfaces}

The developments of the joining geometries and techniques presented herein are motivated by situations arising in the fabrication, installation/assembly, and in- 
service performance of a system for construction of complex curved surfaces from composite skin-and-core panels whose core structure is to be formed (more specifically milled, or at least partially wire-cut) from sheet material of uniform thickness. Since this criterion of uniform thickness before shaping imposes limits on the size of individual panels due to curvature (i.e. flatter panels can be larger, while more curved ones must be smaller), an adaptive subdivision strategy is employed to guide placement of the joints between panels ${ }^{3}$. The subdivision process results in various panel sizes and shapes, and therefore various joint locations, orientations and lengths, which are typically also influenced by primarily in-service considerations such as: 1) joint forces to be withstood, 2) joint movements to be allowed (and/or restricted), 3) weather-resistance requirements, and 4) appearance, as well as by fabrication/installation considerations including: a) joint forming capabilities, b) panel installation methods and sequences, and c) cost. (See Fig. 1.) (CHASZAR 2004, ADRIAENSSENS \& CHASZAR, 2007). While in some cases the application of particular joining details could influence the overall surface's structural and other performances, in general approximate analysis methods can be used during much of the design study to provide more rapid feedback (CHASZAR, 2003, BORGART \& CHASZAR, 2007). Thus, characteristically of situations sufficiently complex to qualify as actual design work rather than 'cookbook' execution of technical procedures, there is present here a potential feedback and tension between 'bottom-up' and 'top-down' influences, which in some cases may even reflect back as far as (usually local) alterations of the main surface geometry (CHASZAR \& COENDERS, 2007). Such an iterative and recursive design process, although in some instances manageable 'manually', generally requires digital computational techniques for effective execution.

The resulting joint paths are notionally straight (e.g. geodesic) in order to reduce joint lengths but generally torqued and usually also curved, according to the needs and opportunities of the specific situation. (NB strictly speaking, they are almost always curved, depending on the plane of projection; only a few rare instances are truly linear). While in some cases the curved joint paths may advantageously be simplified through constraint to a single plane of curvature, this will, however, generally result in the primary joint plane not being normal to either of the panel's external surfaces, nor to its (geometrically, if not mechanically) 'neutral' surface ${ }^{4}$. Figure 2 shows a partial plan of the overall surface, while Figure 3 shows a typical panel section and panel plan.

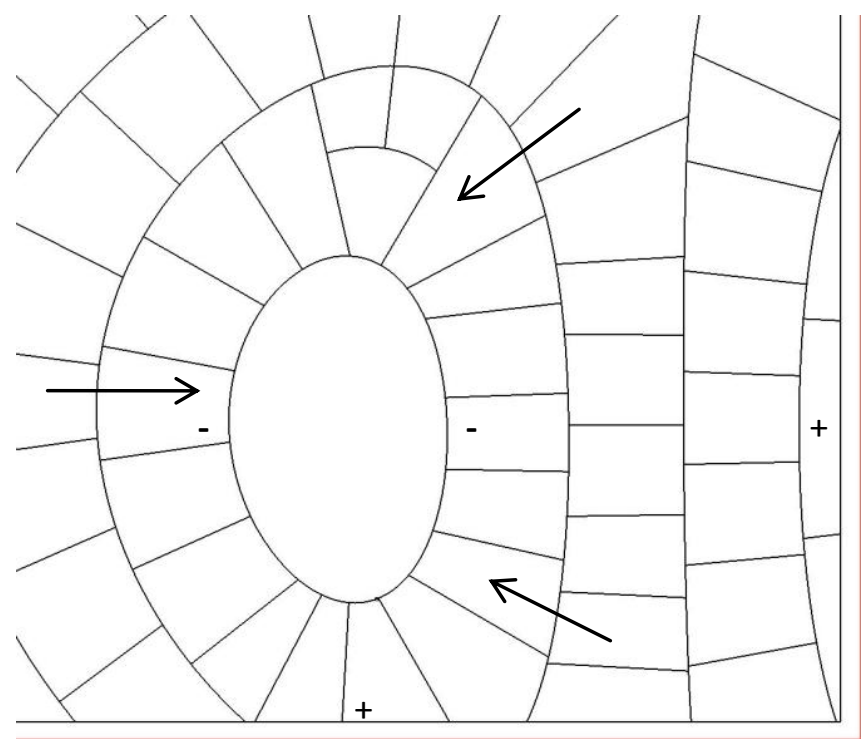

Figure 2. Partial plan of subdivided surface, indicating high (+) and low (-) points, slopes and panel joints. Source: author, 2013.

Joint lines initially are oriented by gradient and isoelevation lines but modified to accommodate nesting where advantageous. Resulting panels may be synclastic or anticlastic in main (or neutral) surface curvatures, and panel thicknesses may vary in both core and skins.

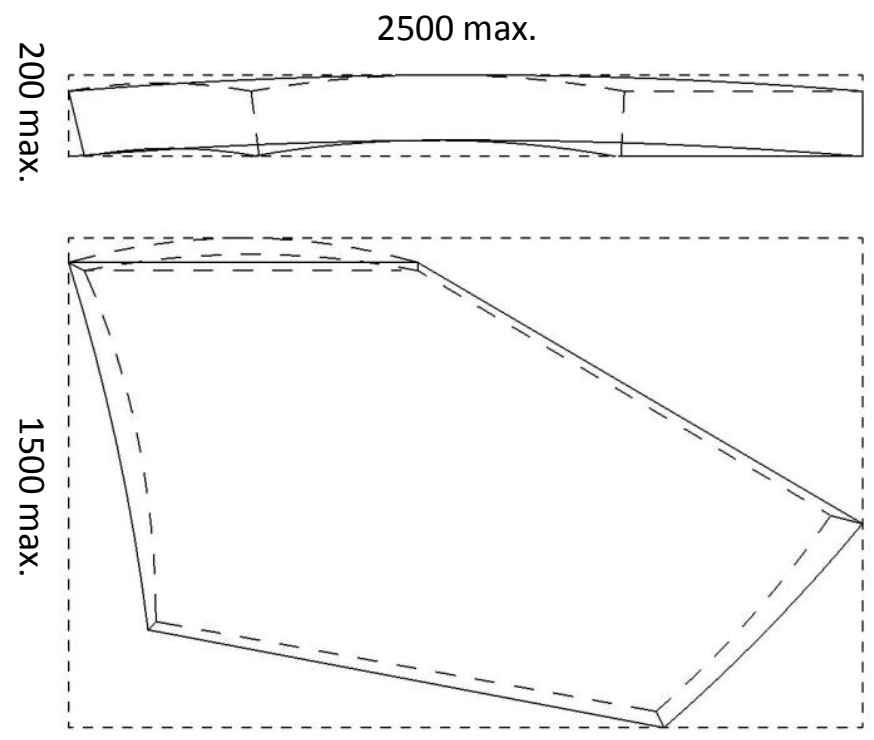

Figure 3. Example panel section (top) and plan (bottom) indicating constraints of uniform (flat, millable) base material thickness and plan dimensions, as well as a few varying possible panel edge (i.e. joint) path trajectories. Source: author, 2013.

Resulting panel weights for the system under study would be under $\sim 180 \mathrm{~kg}$ (the maximum for a completely flat panel with maximal dimensions of $1.5 \times 2.5 \mathrm{~m}$, of which the particular project has none) and average $\sim 100$ 
kg. Actual final panel sizes must balance panel fabrication and joint forming capabilities with transportation and on-site assembly conditions, as well as considering in-service performance issues. The envisioned construction sequence favors placing panels first at the surface perimeter, then working inward -- in contrast with the inverse of this, or with proceeding from one end/edge of the surface to the furthest opposite one. Joints are laid out in a 'radial' (actually gradient-based) pattern in consideration of joint forces and water runoff, although with some local exceptions intended to improve (i.e. reduce) joint quantity and increase surface constructability without unduly compromising in-service performance. Nesting of panel shapes within maximum core material boundaries also plays a role in this layout process, aiming to reduce wastage of core material. Cross-slope joints (nominally horizontal) are detailed with overlaps in some locations to promote ventilation as well as ease installation and fabrication, as mentioned already above and discussed further below.

\section{Detail - joining adjacent panels: joint criteria, types/parameters, evaluation and selection}

In this section we examine in more detail the performance requirements for panel joints in general and also more particularly for the specific example surface. We also look at 1) how various joint detailing options fare with respect to the relevant requirements, 2) how decisions can be made to choose among existing alternatives joint detail designs and/or 3) how additional alternatives can be generated to reach specific goals.

\section{Joint performance criteria and options}

Alternative options for detailing of the panel joints range from geometrically simple to complex and from high- to low-tolerance, each of which have advantages and disadvantages with respect to the performance criteria mentioned above (as well as to others not yet mentioned here). Since the surface to be subdivided into panels is not necessarily formed to optimise structural performance (sacrificing some of this in favor of others relating to ventilation, lighting, acoustics, etc., in keeping with the observations on multi-criterion design above), panel joints may be subjected to a variety of forces and combinations thereof. These include in-surface compression, in-surface tension, in-surface shear, through-surface shear, bending and torsion. As not all joint types are capable of accommodating all stress types, these will limit options to some extent. Also, as not all joints within the given surface are subjected to the same states of stress, joint types by this criterion may vary at different locations.

Joint movements are also to be taken into account, for example as ways to relieve stresses due to thermal expansion and contraction or to surface flexure under imposed loads (e.g. self-weight, live loads, wind, etc.) In general, smaller movements can be accommodated by compression and/or tension in joint materials - thus maintaining closed joints - while larger movements require incorporation of gaps in the joint detailing. Depending then upon factors such as weather resistance, air-tightness and acoustic isolation, joint gaps may need to be covered more or less tightly. Movement capacity in joints is influenced not only by geometry, of course, but also by materials. The extent to which the panels' materials themselves and any additional joint materials can be stretched, compressed, sheared or torqued influences various aspects of their performance both inservice and during construction. Thus, for complex curved surfaces the resulting joints' curvatures in one or more directions normally benefit from being accommodated by formed joint materials which have some capacity for both elastic and plastic deformations, within limits avoiding structural instability. This generally improves their resistance to air- and waterpenetration and also to movements resulting from thermal and other forces, as well as allowing easier installation. (Poured or trowelled joining materials can also be appropriate choices to address these issues.) Joint detailing choices are conditioned also -- especially in terms of movements and forces to be accommodated -by whether panels are to be supported by a substructure or instead be self-supporting (as in the case of the project motivating the present study), and in the latter case especially by the panels' self-weight, imposed loads, and inherent flexibility, elasticity and/or brittleness.

Panel joints' weather resistance requirements - e.g. water-tightness, water-resistance, air-tightness - depend on geographical location (i.e. local climate and weather patterns) (DANIELS, 1997) as well as on project-specific needs resulting from intended use of the (semi-) enclosed spaces, and also on the particular surface position(s) horizontal, vertical, inclined. As with the issues of joint stresses and movements, different requirements and therefore different joint types may apply at different locations, despite the conceptual unity of a surface. In the particular case at hand, water-resistance but not -tightness is required at all but the lowest regions of the surface, and air movement through the joints is also acceptable or even desirable, especially at the higher regions.

For panelised surfaces in general, there arises the issue of using formed panel edges vs. edge inserts vs. separate joining elements (e.g. mullions). While direct machining 
of the panels can in some cases achieve with minimised effort all necessary technical performance requirements, the insertion of panel edge elements set flush with main panel surfaces and edges (or variations with non-flush projections) offer opportunities for simplifying machining and/or other forming operations by allowing the edges to be handled separately. Panel edge inserts whether continuous, intermittent or isolated (i.e. 'point fittings') - for complex curved surfaces/joints generally benefit from digital techniques of design and fabrication, as they often require a high level of precision or at least accuracy in their placement as well as in their shaping. Some panel types and insert types are more forgiving than others, of course. Custom-machined metal pointfitting inserts to laminated glass panels - such as those used in the Macalester Hall project (LOWINGS \& CARPENTER, 2003) and those later more ubiquitously deployed on the worldwide Apple stores -- exemplify high-precision and accuracy types. A simpler mediumlevel approach, also for laminated glass, was taken on the BIPV solar pavilion (CHASZAR, 1999) with surface mounting, adhesives and neoprene substituting for some of the more costly joint forming operations. In contrast homogeneous (or approximately so) panels such as concrete precast ones fabricated and installed to typical construction tolerances represent a point nearer the opposite ends of these spectra. The case of sandwich panels at hand here is a somewhat curious amalgam, as the interplay of relatively thin skins and thicker core mean that the degrees of accuracy and precision required vary greatly with the type of joint detailing chosen, and with which joining materials engage which panel materials, as discussed further below.

Custom-made edge inserts or other joining elements such as mullions and transoms, or hubs and rails encompass in themselves already a very wide range of options in shape, material and capabilities. One prominent example of millable synthetic material shaped to meet the needs of particular projects and/or of location/position-specific geometry and performance has been successfully developed and installed as detailing of glass panel supports for the complex curved surfaces of the Hungerburgbahn stations in Innsbruck by Design-toProduction (SCHEURER, 2010). Many other types of continuous or discrete edge elements fitted adjacent to or within the panel edges have been designed, as enumerated and described partially below, and of course many many further variations and some new types are still possible. On the other hand selection of appropriate materials, joint types and panelisation patterning to ensure a constructable sequence of installation also suggests that the panel edge itself be milled in some cases, if proper adhesion of skins and adequate manipulability/accessibility for $\mathrm{CNC}$ operations due to panel shape, size and weight are ensured. In yet another set of variations, there arises also the possibility of forming/shaping composite panel skins and/or core separately from each other, again with proper attention to de-bonding issues.

Much of current architectural fashion still prefers detailing which preserves the smoothness of surfaces ${ }^{5}$, and therefore formed edges and/or inserts, but projecting joining elements (e.g. flanges, standing seams, etc.) may also be appropriate technically and otherwise, as can overlaps of panel edges. Indeed, many issues of fitting tolerances and weather-resistance can be addressed by time-tested and/or novel techniques of overlapping, though these present additional challenges to computation of panel shapes, especially in the event of double-curvature panels (POTTMANN et al., 2007). Nevertheless, the simplification where applicable of many sub-surface edges' detailing by these means can amply justify the effort. Here again digital computational capabilities can make feasible designs and fabrication of details which otherwise would not be.

Still further opportunities are presented by cold-forming with bending and/or twisting -- especially of panel edge inserts or other fittings, but in some cases even of panels themselves -- within suitable ranges of material and geometric stiffnesses and of other material properties (e.g. elasticity, plasticity, reserve strength in elastically and/or plastically deformed states, etc.) (CHASZAR 2002). This approach can simplify digital forming operations and expand the range of assemblable geometries. While much of such forming is still done with pre-digital techniques, in some instances $\mathrm{CNC}$ bending jigs or even robotics may be employed, thereby achieving lower costs and/or higher precision and accuracy, depending on the specific market and available skills.

Another important general issue common to various projects concerns (semi-)fixing of joints 1) mechanically, 2) by fit, 3) by adhesive, or 4) by combinations thereof despite the rule-of-thumb favoring non-combination due to uncertainty of work sharing. Snap-fitting of edge inserts and even of panels (via their edge detailing) can reduce the labor needed for fixing of edge inserts, which might otherwise largely if not entirely negate the gains realised in machining and handling the edges separately. Adhesives with gap-filling capabilities can ease some fabrication and installation hurdles, which again - like overlapping - may be contrary to the prevailing digitally/computationally-driven appetite for precision, yet do provide necessary relief under practical project pressures. 


\section{Joint types, characteristics and variable parameters}

Some specific examples of joint detailing options examined in connection with complex curved composite panels and reflecting the observations above include the following types, along with their most important characteristics:

- 'butt' - This basic joint type is formed with a planar cut, or more often with other ruled surface cuts due to the changing orientation of the panel surfaces' normal vector(s). Its constructibility depends largely on gap size, which can be adjusted to accommodate sequential panel placement, though this will usually require some gapfilling materials or fittings to be placed subsequently, directions tends to restrain movement. Weathering performance is as above.

- 'spline' - This is a butt joint with a continuous (or sometimes interrupted) relatively thin insert (Figure 6) giving greater restraint and force transfer than the plain butt or dowels, but it also has limitations as above, only more so. The spline piece/material normally needs to be flexible enough to allow it to follow panel curvatures, if milling or moulding it to shape are to be avoided, and especially to allow some deformation during installation. If suitable panel shapes and assembly sequence can be designed, the spline does offer better weather resistance than the two preceding types due to a longer path for air, water or dust impinging the joint. In some cases

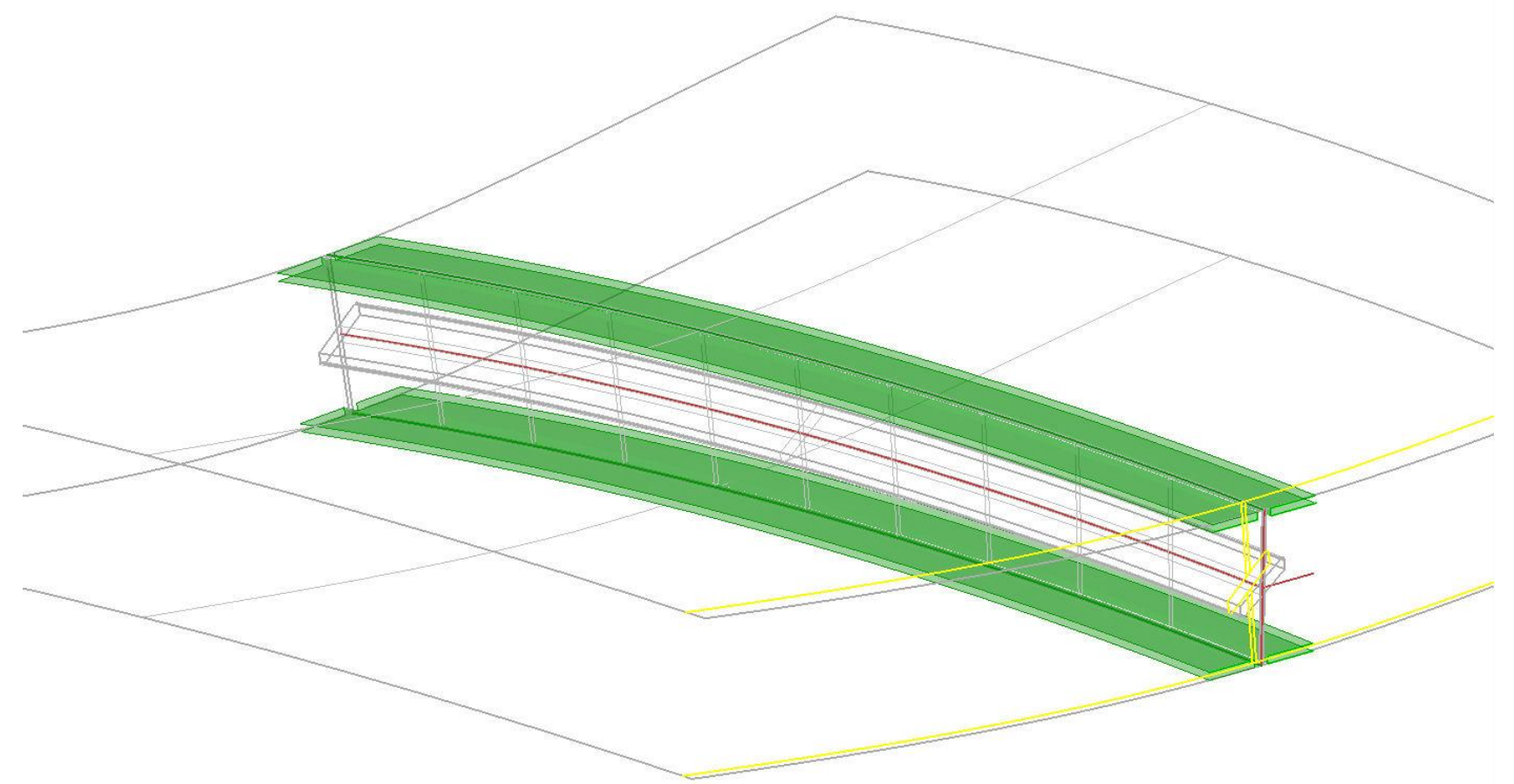

Figure 4. General view of representative panel joint following curved, torqued path. Joint details' geometry may conform or simplify these conditions to reduce forming complexity and/or aid installation. Source: author, 2013.

unless open joints are acceptable and a supporting substructure is present. This joint type can accommodate some movement but only limited force transfer via friction and/or adhesion. Its weathering performance depends on the qualities of the gap-filling material/fitting, if any. (See Figure 5.)

- 'butt' with dowels - This variant of the basic butt joint has greater limitations on constructibility due to installation sequencing, which can in some cases be alleviated by insertion - and possible later covering - of dowels from a panel surface rather than edge. It can accommodate some movement if no in-surface compression is to be resisted except at the limit of joint gap or filling; however, placement of dowels in multiple installability can be improved if we consider alternatives to placing splines perpendicular to panel edges and parallel to surface(s), instead inclining them to accommodate panel placement.

- 'bulb' - This resembles a spline joint with one side fixed, and the other normally sliding along the joint axis for installation (if flexible enough for the curved path), or 'snap fit' to allow insertion but not extraction. The flexibility needed for installation in either case partially reduces force transfer transverse to the panel surface, as with the spline. Also as with the spline it forms a barrier to air, water and dust, though subject to grit accumulation reducing sliding movement if no additional sealant is provided. 
- 'double bulb' - As the bulb above but inserted to grooves in both panels, also requiring a relatively flexible stem and again providing a partial barrier. Note that while a circular cross-section is common for extruded bulb profiles, other shapes may be preferable if they are to be milled. In any event the double-bulb is an edge insert, while treating the single bulb as 'integral' requires using a higher density core material than would usually be needed, or at least changing core materials near the panel edges. (This, however, further restricts the geometry of joint paths along which the bulb can slide.)

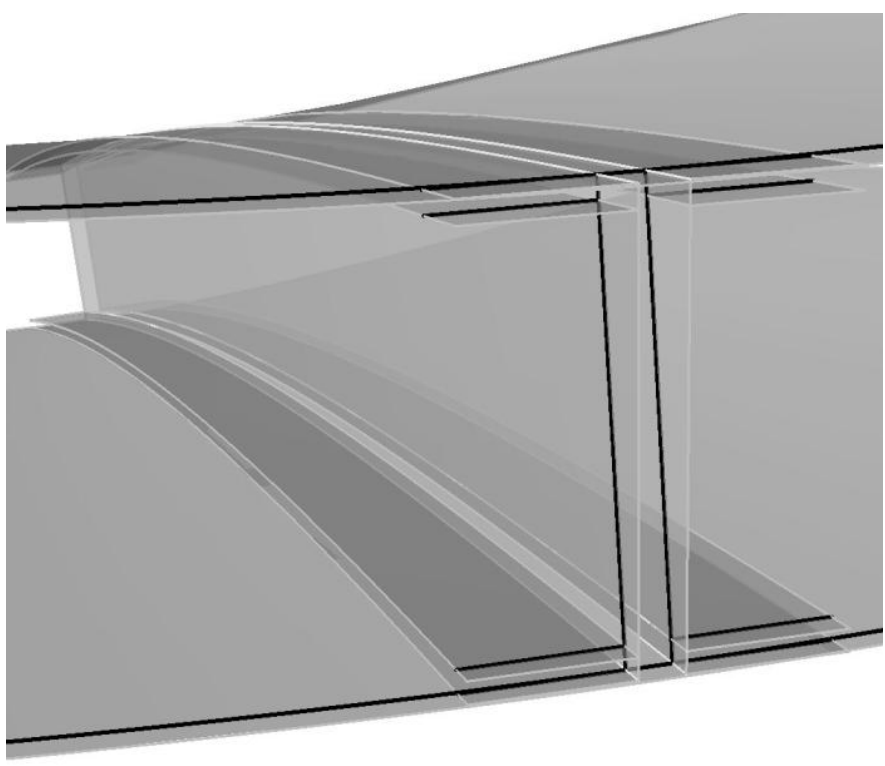

Figure 5. Simple butt joint with torqued, ruled mating surface. Most appropriate for open (possibly surface-sealed) joints with indipendent panel supports, or low panel displacement and force transfer via adhesive gap filler. Source: author, 2013.

- 'flange' - Joints with flanges comprise a large group with various sub-types, projecting one or both sides, integral (e.g. standing-seam) or applied as fittings. They have limitations on geometry due to complex curvature, normally requiring deformability and/or fitting tolerances, in some cases (filled) gaps if installed intermittently. For skin-and-core composites the most direct means of forming a flange is to cut back the core and one skin, leaving the other skin to project and engage a cut-back skin over the core of the adjacent panel. Depending on panel shapes and installation sequencing, however, it may be preferable to hold back skins on one or both surfaces of both panels, resulting in something like a butt joint with flush covers. In such cases throughsurface force transfer relies on very good bonding of the skins to the core. (Figure 7.) Good weather-resistance and minimal joint visibility can be achieved if only little or no joint movement needs to be allowed. CNC milling to form such joints is fairly simple, little more involved than making butt joints. Joints with applied flanges, on the other hand, are even simpler to fabricate and install, though at the price of greater visibility (not always a detracting factor, however, especially if panel surfaces are not visible).

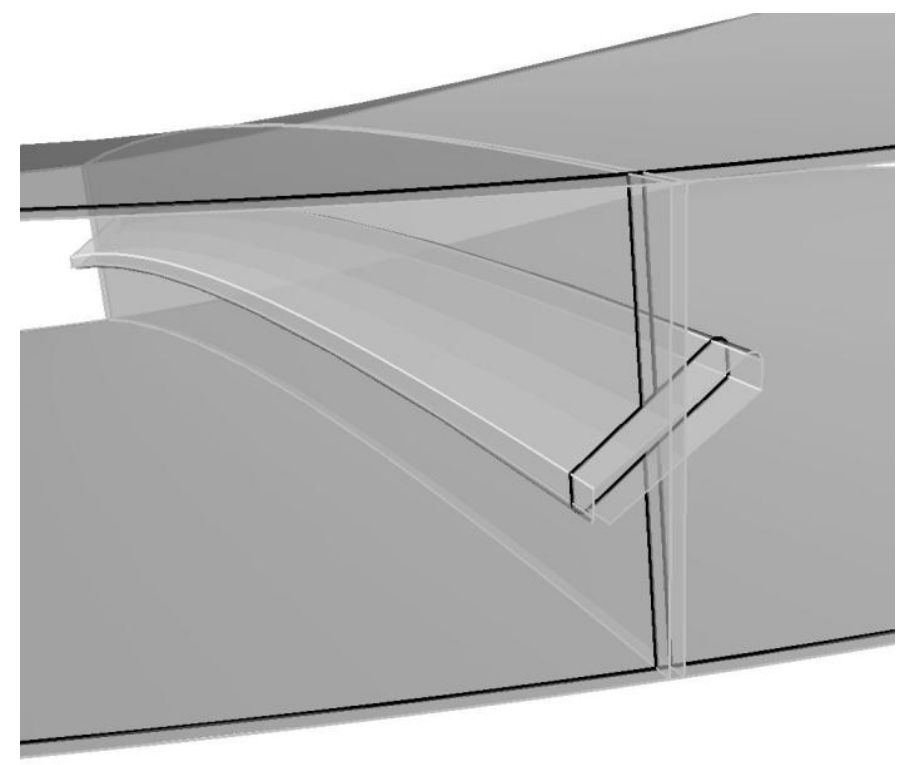

Figure 6. Spline joint with machined keyways and spline inclined to improve installability. Source: author, 2013.

- 'plug' or 'butterfly' - These joints are a hybrid of dowel and bulb types, with discrete rather than continuous headed projections linking the panels. Installation normally requires that the cavities to receive these be open from above and/or below. These joints can provide good tension and some in-surface shear capacity. Their weather-resistance is comparable to the butt and dowelled butt joint. As with the bulbed joints, fabrication by milling may suggest head geometries other than the rounded ones obtainable by casting or extrusion.

- 'jigsaw' - This works similarly to the type above, but with a continuous series of interlocking projections for in-surface shear by mechanical/bearing transfer, not by adhesion/friction. Draft angles of the interlocking surfaces need to be carefully considered, otherwise joint gaps and/or panel flexibility are needed to enable assembly of adjacent panels.

- 'shingle' - This joint type is characterised by open or closed overlaps at panel edges. This results in relatively easy constructibility, and it also can accommodate ventilation and/or quite large movements, when joints are open. Force transfer is somewhat limited, however, in comparison with more continuous arrangements.

Given the foregoing partial enumeration of geometries and performance capabilities, we can turn to the matter of selecting and refining joint types. 




Figure 7. Flanged joint with overlapping skin layers and machined seat in core material. Joint parameters include skin projection/cutback lengths and seat width and angle, in addition to the usual choices regarding joint cut angle and path. Source: author, 2013.

\section{Evaluation and selection of joint types and parameters}

To the extent that the joint details listed (and others as well) are distinct, already developed types, a rational selection among them could proceed along MCDA lines, with scoring of each alternative in respect to each relevant performance criterion leading to identification of one or more 'best' options. To the extent that some of the criteria may not be possible to precisely quantify, a looser scoring system may be needed than that provided by simulation/calculation-based analyses, which again fits within the general framework of MCDA. Such an approach also accommodates other, 'soft' factors for which no valid mathematical evaluation is known. On the other hand, those parameters of the joint details which are inherently numerical or mathematically representable especially those which are continuously variable, such as the dimensions and/or quantity of details' various subfeatures - can be subjected to an iterative optimisation-based process, as with MCO. In these instances new choices can be generated in a goal-driven (or more exploratory) way, rather than sticking only to pre-designed alternatives (as when selecting from a catalog, for example, with no option for customisation.) The likelihood, if not necessity, that the joints will be produced with $\mathrm{CNC}$ fabrication technology due to the complexity of the surface geometry and/or joint geometry increases greatly the scope for such continuous-variable optimisation, in comparison with using standardised fittings.
Evaluation of and selection among the various joint options in the current state of the present project's design development is in this case based largely on experience and exploratory motivations. However, given sufficient information and resources, it would be of interest to also make a more comprehensive comparison via 'fitness functions' or other relevant measures of performance/quality/value to help finalise or further refine choices. Simulations or other calculations may provide additional data for MCO/MCDA analyses, giving a set of joint detailing choices displaying Pareto Optimality most likely, from which final choices could be made on the basis of further judgement. We can here anticipate (Figure 8) what a hypothetical, indicative Pareto surface of joint strength/stiffness, weather resistance and constructibility at constant panel (and joint) material choice, joint layout/pattern and cost would look like - noting that cost would be an installed/initial or modified lifecycle cost ${ }^{6}$.

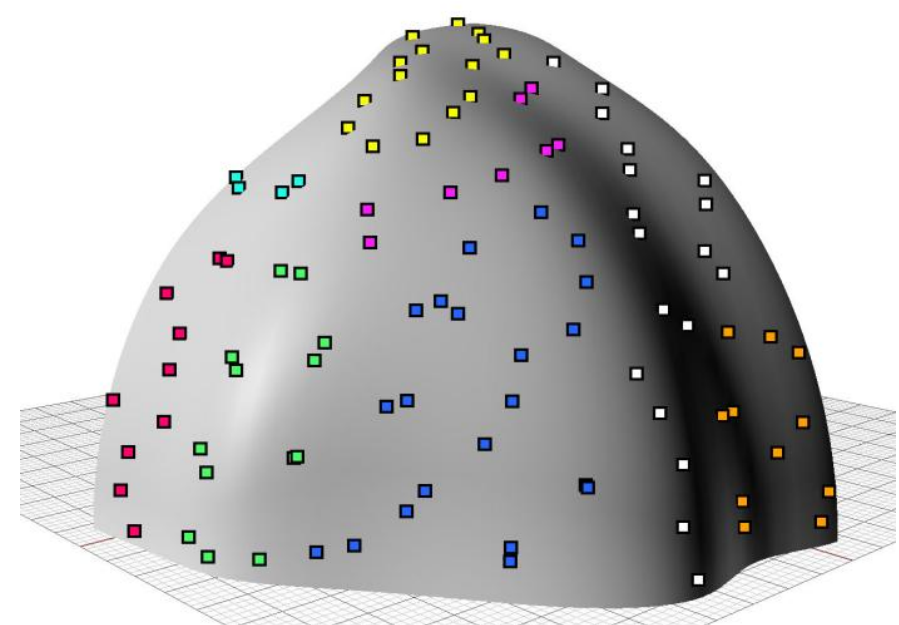

Figure 8. Hypothetical Pareto-Optimal surface of joint types (colors) and variations of their parameters, plotting three performance dimensions while holding others constant. Source: author, 2013.

In this we observe a relative sparseness of points, due to there being distinct and relatively few joint types rather than a continuum of accessible alternatives (vis MCDA); however, some localised 'spread' is expected due to the variability of joint geometry parameters for some types, and thus some potential for continuous 'blending' between some types. Further 'blending' lending itself to MCO methods might also be contemplated where joint materials are also composites, with 'tunable' material properties rather than distinct, discrete values from one material type to the next. In the event that such (sub)optimisation (tuning) is possible for joint detail parameters, not just joint layout parameters, then there is most likely a need for design aids and decision support in relation to making sense of the often complex relationships between inputs and outputs, and between 
input parameters themselves. For this techniques of 'multivariate interactive visualisation' are applicable. These can help to make a more intelligent use of (semi-) automated design space exploration methods and tools by revealing where parameter correlations exist, suggesting when parameter ranges and/or step sizes should be expanded or contracted, and perhaps even indicating when new parameters may need to be considered (CHASZAR 2013, CHASZAR et al., 2014). As pointed out before, the criteria used for selecting among these given or generated "equally good" options remain outside the direct comparison, and they may be explicit or implicit.

\section{Discussion and conclusions - less is more, more or less?}

In summary of the above: the foregoing has described issues of joint detailing - including design options (e.g. joint types and parameters) and methods of resolution (e.g. MCO/MCDA, Pareto-optimality, multi-variate interactive visualisation) - in general and for the more specific case of composite skin-and-core panels resulting from subdivision of complex curved surfaces, all in relation to the application of digital techniques. The joint details described as well as the parameters affecting the choices among them are to varying extents applicable also to other types of panels (e.g. homogeneous) and other types of surfaces (e.g. flat, or less complexly curved ones). The methods of resolution are even more generally applicable, of course, to all kinds of multi-criterion optimisation and/or decision analysis (MCO and MCDA) situations. Multivariate interactive visualisation in particular, and Interactive Visual Analysis (IVA) and Visual Analytics (VA) in general, are areas of already mature but also still active and ongoing research which can be brought to bear on complex issues of architecture, engineering and construction such as this specific question of detailing complex curved panel joints. However, further work is needed to enable quantification and/or other comparison of joint performance dimensions such as weather-resistance and constructibility to be incorporated within such techniques for analyses of options.

In contrast with 'futuristic' design proposals for constructive schemes represented, and in some cases generated, through digital techniques - often characterised in their 'details' by features such as hair-thin lines on surfaces, bio-network-like tendrils, or amorphous (perhaps nano-) goo - the present contribution has focused on mechanical (not molecular) level features realisable with current, commercially viable fabrication techniques and responding to known performance requirements ${ }^{7}$. However, looking somewhat ahead, it can be interesting to speculate on how joint detail types such as those we have been examining and others may evolve, and on how methods of evaluation and generation themselves may also evolve.

We can envision, for example, a process in which joint types for particular situations are selected on the basis of affordances, at least some of which can be expressed in shape-based ways. For two examples: 1) water runoff and consequent water-tighness/resistance requirements can be related at least partially to the geometrically influenced factors (e.g. slope, catchment area, air pressure potential, joint orientation) affecting the joint at a particular location; and 2) shear forces present at a joint typically suggest the need for detailing to include bearing surfaces - whether of joined elements themselves, or of intermediating fasteners, etc. -- suitable in size and orientation (for given material choices) to transfer those forces. To the extent that shapes in combination with material properties can satisfy such requirements, details could be developed as amalgamations of shapes+materials which deliver the desired performance, conceivably without reference to existing joint types (although probably still arriving at quite similar designs in most cases.) Such approaches are already maturing in mechanical design research and may find their way thereby relatively easily into architectural applications as well. This becomes especially interesting when features selected for one purpose turn out to serve more than one, either immediately or with some further tweaking.

Recognising that the foregoing approaches to detail development and selection are heavily performancedriven, based on rationalistic evaluation of explicitly described options with respect to explicitly described performance requirements/goals, we may also consider other approaches appropriate or even necessary due to factors such as implicit goals, limited information, knowledge or skills, and/or an emphasis on the expressive capacity of detailing, among others. Such other approaches may be pursued for quite practical reasons such as fostering innovation through constraint relaxation, the temporary acceptance of 'bad ideas' (SOSA \& GERO, 2013) for path-dependent exploration processes, and the support more generally of 'expandable rationality' (HATCHUEL, 2002) which can even include redefining the 'problem' that a joint and its features should address.

We should bear in mind that even a strictly technically driven approach may also be subjective to some extent due to both the potential inexhaustibility of possibly relevant criteria and the need to select among "equally good" options. Thus, while in theory an extensive comparison of various joint types and parameters is required, in practice this is typically resolved by counting 
some criteria as substantially more important than others, thereby limiting the latter's influence on detailing choices or indeed even obviating the need to evaluate those lesser factors. Such obviation does not, however, necessarily eliminate or limit the effects of those factors in fabrication, assembly and/or service - it only ignores them. Frequently joint layout appearance, machinability/constructibility, strength/stiffness and weather resistance (if applicable) are prioritised, with resulting options compared on the basis of cost and the choice adjusted accordingly, or further modifications made if cost cannot be adequately balanced with alreadygenerated options. The full-scope rational analysis is thus circumscribed to fit available resources (aka 'satisficing' HERBERT 1957). One significant potential of introducing digital techniques in design and analysis is that the process can be less circumscribed, resulting in better designs and realisations - such as through the incorporation of more expert knowledge into design decision support tools (VOSS \& OVEREND, 2012) though there is always the possibility that due to commercial pressures this increased power of generation and evaluation will only be used more to compress project schedules rather than to improve the quality of designs. There is also the issue of possibly restricting the factors to be dealt with to computable ones, which may merely shift, or even contract, the breadth of design exploration (CHASZAR, 2012).

We have also seen that while panel joint detailing encompasses a relatively small number of factors and degree of complexity in comparison to the situations prompting identification of 'wickedness' in problems of design or policy, the interrelatedness and lack of a clear hierarchy among means and goals of joint design - as well as the lack of precision of relevant criteria such as 'constructibility', or even 'weather resistance', for example - mean that joint design in architecture normally involves 'satisficing' and remains as much art as science. Thus, it is possible that in many cases stepping back from the maximum achievable limits of speed, size, precision, complexity, etc. will achieve better results overall both in the present and in the longer term.

In conclusion we may suggest that even within the microcosm of detailing - though vastly simpler than the design of entire buildings or cities, yet still presenting a challengingly intricate interrelation of relevant factors the conviction of a single 'best' solution may have value as a rhetorical, motivational device but not as rational decision. Instead, we need to recognise the partiality of chosen 'solutions' and remain capable of revising them when needed, in which digital techniques can play an important role. Such awareness of situations and exercise of judgment may be the most important respects in which craft remains operative within the otherwise rationalising realm of computation.

\section{Notes}

(1) The absence or presence, number, location, distinctiveness, etc. of attractors in such recursive processes would be an interesting study in its own right and falls outside our present scope, but suffice it to say that even if convergence is not apparent, the process may be terminated at some acceptable level of equilibirium.

(2) Though some would limit the term 'performance' to quantifiable aspects only, 'soft' factors and other unquantifiable evaluations of quality/desirability/fitness remain important considerations.

(3) Details of this subdivision approach - which comprises various methods including [Adriaenssens and Chaszar 2007] - are beyond the scope of the present paper.

(4) Again, we take here the case of complex curved surfaces in general; specially constrained surface geometries may in some cases further simplify joint geometries.

(5) It remains open to question whether this is due just to the technical expedient arising from introduction and persistence of digital techniques originating in modelling and animation for entertainment, or also to other influences such as: a fascination especially of younger digitally oriented designers with vehicles and human forms, the late modernist adoption of minimalthickness curtainwall cladding as a way to maximize usable floor areas, or a symbolic association of smoothness with contemporaneity or even with the future. In any case, it tends to introduce a number of technical difficulties, sacrifice some advantages found in more articulated surface joining, and favor fantasies of seamlessness and ease.

(6) "Lifecycle cost" is a questionable criterion due to the unpredictability of future costs of maintenance, energy, etc., but some assessment within a reasonable time horizon can be helpful to avoid options which are only economical in first-costs.

(7) Thus, no proposal here for thousands of special-alloy, laser-sintered fasteners or fittings, for example, nor for genetically modified mycelia bindings, though both of these are conceivable. 


\section{References}

ADRIAENSSENS, S.; CHASZAR, A. (2007) Dynamic relaxation and related methods for subdivision and panelling of complex curved surfaces, working paper.

BORGART, A.; CHASZAR, A. (2007) Computational methods for analysing and decomposing complex curved structures, in Proceedings of International Association for Shell and Spatial Structures (IASS), IUAV, Venice.

CHASZAR, A. (1999) BIPV exhibition pavilion: multi-functional use of glass, in Proceedings of Glass in Building, CWCT, Bath. pp. 127-134.

CHASZAR, A. (2002) Parametric design of a wave-form roof. Presentation at Bentley International User Conference, Atlantic City.

CHASZAR, A. (2003) Bridging the gap with collaborative design programs. Architectural Design v.73, no.5, pp. 112-118.

CHASZAR, A. (2004) O-Design Technical Report: Design development and detailing of complex curved building roofs and other surfaces - Queens Museum of Art Expansion.

CHASZAR, A. (2006) ed. Blurring the Lines: Computer-aided Design and Manufacturing in Contemporary Architecture, WileyAcademy. 206 p.

CHASZAR, A.; COENDERS, J.L. (2007) Parametric and associative strategies for shell and spatial structures, in Proceedings of IASS, IUAV, Venice.

CHASZAR, A. (2012) Beyond BIM: reflections and research on design communication via digital building models, in P. Deemer and P.G. Bernstein (eds.) BIM in Academia, Yale School of Architecture, pp. 42-53.

CHASZAR, A. (2013) Finding rhythms in a tempest, in J. Burry (ed.) Designing the Dynamic, RMIT, pp. 118-123.

CHASZAR, A.; TURRIN, M.; von BUELOW, P. (2014) Multivariate interactive visualisation, forthcoming.

DANIELS, K. (1997) Technology in Ecological Building: the Fundamentals and Approaches, Examples and Ideas, Birkhauser.

HATCHUEL, A. (2002) Towards design theory and expandable rationality. Journal of Management and Governance v.5, no.3-4, pp. 260-273.

LOWINGS, L.; CARPENTER, J. (2003) Constructing the ephemeral: innovation in the use of glass, in A. Brookes, D. Poole (eds.) Innovation in Architecture, Routledge.

POTTMANN, H.; ASPERL, A.; HOFER, M.; KILLIAN, A. (2007) Architectural Geometry. Bentley Institute Press.

SCHEURER, F. (2010) Materializing complexity, Architectural Design, v.80, no.4, pp. 86-93.

SENNETT, R. (2008) The Craftsman. Allen Lane.

SOSA, R.; GERO, J.S. (2013) The creative value of bad ideas, in Proceedings of CAADRIA, NUS, Singapore. pp. 853-862.

VOSS, E.; OVEREND, M. (2012) A tool that combines building information modeling and knowledge based engineering to assess façade manufacturability, in O. Englhardt (ed.) Advanced Building Skins, TU Graz. 